\title{
Antimycobacterial Activity, Synergism, and Mechanism of Action Evaluation of Novel Polycyclic Amines against Mycobacterium tuberculosis
}

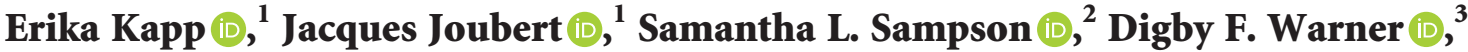 \\ Ronnett Seldon $\mathbb{D},{ }^{4}$ Audrey Jordaan $\mathbb{D}^{3},{ }^{3}$ Margaretha de Vos $\mathbb{D},{ }^{2}$ Rajan Sharma $\mathbb{D},{ }^{1}$ \\ and Sarel F. Malan ${ }^{1}{ }^{1}$ \\ ${ }^{1}$ School of Pharmacy, Faculty of Natural Sciences, University of the Western Cape, Cape Town, South Africa \\ ${ }^{2}$ DSI/NRF Centre of Excellence for Biomedical Tuberculosis Research, SAMRC Centre for Tuberculosis Research, \\ Division of Molecular Biology and Human Genetics, Faculty of Medicine and Health Sciences, University of Stellenbosch, \\ Cape Town, South Africa \\ ${ }^{3}$ SAMRC/NHLS/UCT Molecular Mycobacteriology Research Unit, \\ DSI/NRF Centre of Excellence for Biomedical Tuberculosis Research, \\ Department of Pathology and Institute of Infectious Disease and Molecular Medicine, Faculty of Health Sciences, \\ University of Cape Town, Cape Town, South Africa \\ ${ }^{4}$ H3D Drug Discovery and Development Centre, Department of Chemistry, University of Cape Town, Cape Town, South Africa
}

Correspondence should be addressed to Erika Kapp; ekapp@uwc.ac.za

Received 11 January 2021; Accepted 24 May 2021; Published 21 June 2021

Academic Editor: Heng Yen Khong

Copyright (c) 2021 Erika Kapp et al. This is an open access article distributed under the Creative Commons Attribution License, which permits unrestricted use, distribution, and reproduction in any medium, provided the original work is properly cited.

Mycobacterium tuberculosis has developed extensive resistance to numerous antimycobacterial agents used in the treatment of tuberculosis. Insufficient intracellular accumulation of active moieties allows for selective survival of mycobacteria with drug resistance mutations and accordingly promotes the development of microbial drug resistance. Discovery of compounds with new mechanisms of action and physicochemical properties that promote intracellular accumulation, or compounds that act synergistically with other antimycobacterial drugs, has the potential to reduce and prevent further drug resistance. To this end, antimycobacterial activity, mechanism of action, and synergism in combination therapy were investigated for a series of polycyclic amine derivatives. Compound selection was based on the presence of moieties with possible antimycobacterial activity, the inclusion of bulky lipophilic carriers to promote intracellular accumulation, and previously demonstrated bioactivity that potentially support inhibition of efflux pump activity. The most potent antimycobacterial demonstrated a minimum inhibitory concentration (MIC 99 ) of $9.6 \mu \mathrm{M}$ against Mycobacterium tuberculosis H37Rv. Genotoxicity and inhibition of the cytochrome $b c_{1}$ respiratory complex were excluded as mechanisms of action for all compounds. Inhibition of cell wall synthesis was identified as a likely mechanism of action for the two most active compounds (14 and 15). Compounds 5 and $\mathbf{6}$ demonstrated synergistic activity with the known Rv1258c efflux pump substrate, spectinomycin, pointing to possible efflux pump inhibition. For this series, the nature of the side chain, rather than the type of polycyclic carrier, seems to play a determining role in the antimycobacterial activity and cytotoxicity of the compounds. Contrariwise, the nature of the polycyclic carrier, particularly the azapentacycloundecane cage, appears to promote synergistic activity. Results point to the possibility of combining an azapentacycloundecane carrier with a side chain that promotes antimycobacterial activity to develop dual acting molecules for the treatment of Mycobacterium tuberculosis. 


\section{Introduction}

The progressive development of resistance to various chemotherapeutic agents used in the management of infectious diseases presents a serious problem in global public health. Tuberculosis (TB) has re-emerged as one of the most concerning communicable diseases of our time. The notoriously complex structure of the mycobacterial cell wall and the abundance of efflux pumps (EPs) in Mycobacterium tuberculosis limit the intracellular accumulation and antimycobacterial efficacy of numerous antimicrobial classes successfully utilized against standard bacteria [1-4]. Treatment and control of the TB epidemic is further complicated by the development of multidrug-resistant (MDR) and extensively drug-resistant (XDR) strains. Current TB treatment regimens thus comprise a combination of antimycobacterial agents which, as drug resistance develops to the various first-line agents, not only decrease in efficacy and patient acceptability, but also increase in required treatment duration and toxicity.

Based on their mechanisms of action (MOAs), available antimycobacterial drugs can be broadly categorized into three classes: those which disrupt cell wall integrity (e.g., isoniazid, ethambutol, ethionamide, and cycloserine, as well as the experimental 1,2-ethylenediamine, SQ109 [5]); those that limit the energy available for cellular processes (e.g., pyrazinamide and bedaquiline [6]); and compounds that inhibit normal cellular functionality by inhibiting or corrupting biosynthesis of essential macromolecules, cofactors, and metabolites (e.g., the rifamycin, fluoroquinolone, aminoglycoside and oxazolidinone class antibiotics, and para-aminosalicylic acid [7-9]). Reduced susceptibility to these antimycobacterial agents commonly results from spontaneous mutations in the genome of mycobacteria with selective survival fueled by suboptimal exposure to, or reduced intracellular accumulation of, active moieties $[10,11]$. EPs, in particular, have been shown to play a role in inadequate accumulation of drugs within the mycobacterial cell [12]. Depending on the particular mechanism by which a drug's action is overcome, resistance may be specific to a particular molecule or, more alarmingly, result in reduced sensitivity to various drugs that target a particular cellular process (e.g., cell wall synthesis) or act as substrates for an overexpressed efflux pump.

Strategies that improve the accumulation of antimycobacterial compounds within the mycobacterial cell would likely increase the efficacy of the particular compound, but would also minimize genetically encoded resistance aided by subinhibitory chemotherapeutic exposure [13]. Approaches that may promote intracellular accumulation of chemotherapeutic agents could include increasing cell wall permeability [14], facilitation of passive transport of drugs into the cell, alteration of the chemical structure of molecules to reduce their predisposition to efflux [15], as well as direct efflux pump inhibition through the utilization of EP inhibitors [16].

As part of a research project designed to evaluate the possibility of modulating drug resistance and increase accumulation of active moieties in $M$. tuberculosis, a series of polycyclic cage compounds were selected from the University of the Western Cape School of Pharmacy compound library to be evaluated for antimycobacterial and efflux pump inhibitory activity in $M$. tuberculosis. The polycyclic cage derivatives included in this study demonstrate, amongst others, $L$-type calcium channel and $N$-methyl-D-Aspartate (NMDA) inhibitory properties [17] and feature a lipophilic scaffold likely to improve the barrier permeability of selected structures. Subsequent research demonstrated the ability of polycyclic amine derivatives (particularly compounds 3, 5, and 11, Figure 1) to modulate antimalarial drug resistance [18-20]. Criteria for the selection of compounds to include in this study were therefore the presence of moieties with possible antimycobacterial activity, and the inclusion of bulky lipophilic carriers to promote compound accumulation within the mycobacterial cell, as well as biological effects which may promote resistance reversal activity. The possession of potential ion channel inhibitory properties, which may directly or indirectly inhibit efflux pump efficacy $[21,22]$, was an important consideration in compound selection.

Here, we report the antimycobacterial activities of the series of selected compounds and a preliminary investigation into possible MOAs of the active molecules. We also describe a possible modulation of Rv1258c efflux pump activity as indicated by synergistic activity with spectinomycin, a known substrate for the mycobacterial Rv1258c efflux pump [15].

\section{Results and Discussion}

The compounds selected for the study were classified into three categories based on the nature of the polycyclic cage scaffold. Figure 1 shows the oxapentacycloundecane class (compounds $\mathbf{1}$, $\mathbf{2 , 4}$, and 8), the azapentacycloundecane class (compounds 3, 5, 6, and 7), and adamantane class (compounds 9-16). Synthesis of compounds 1-15 (Figure 1) is described in detail in previous publications by our group, as indicated in Table 1. Compound 16 was purchased from Sigma-Aldrich ${ }^{\circledR}$.

2.1. Antimycobacterial Activity. Minimum inhibitory concentrations $\left(\mathrm{MIC}_{99}\right)$ of the compounds (Table 1) were determined in nutrient-rich and nutrient-poor media types by the broth microdilution method utilizing an $M$. tuberculosis $\mathrm{H} 37 \mathrm{Rv}$ reporter strain expressing GFP [29-31]. Compounds $3,5,7,11,14$, and 15 showed antimycobacterial activity with $\mathrm{MIC}_{99}$ values ranging from $9.6 \mu \mathrm{M}$ to $82.2 \mu \mathrm{M}$ (Table 1 ); these were selected for further analysis to determine the possible MOA.

Interestingly, during $\mathrm{MIC}$ determination, compound $\mathbf{5}$ showed activity in the GAST-Fe minimal medium, comprising glycerol, alanine, salts, iron, and Tween80, but not in the standard Middlebrook 7H9 albumin-dextrose-catalase (ADC) medium. This difference may point to the binding of the compound by albumin [22], interference of catalase in the MOA, or an MOA that has an impact on the ability of the bacillus to use glycerol as the primary carbon source [32]. In contrast, the activities of compounds 7 and 14 were much more pronounced in standard 7H9-ADC, while compounds 3 , 11, and $\mathbf{1 5}$ showed comparable potencies in both media types.

An interesting observation pertaining to the structures of the compounds versus the observed antimycobacterial activity was the differences in MIC for the structurally similar 


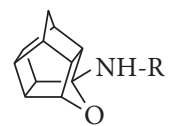

$\mathrm{R}=1,2,4,8$

$1,5,9$<smiles>CCc1ccccc1</smiles>

2,10<smiles>CCc1ccc([N+](=O)[O-])cc1</smiles>

3<smiles>CCCNS(=O)(=O)c1cccc2c(N(C)C)cccc12</smiles><smiles>CCCN1CCN(Cc2ccccc2)CC1</smiles>

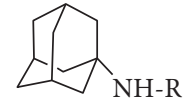

$$
\mathrm{R}=9-16
$$

11<smiles>CCCCNS(=O)(=O)c1cccc2c(N(C)C)cccc12</smiles>

13<smiles>CS(=O)(=O)c1ccc(C(=O)NC23CC4CC(CC(C4)C2)C3)cc1</smiles>

14<smiles>CCCCNc1ccc([N+](=O)[O-])cc1[N+](=O)[O-]</smiles>

15<smiles>CCCCn1cc2ccccc2c1C#N</smiles>

FIGURE 1: Structures of evaluated compounds grouped according to the respective lipophilic scaffold.

Table 1: Antimycobacterial activity and cytotoxicity data.

\begin{tabular}{lccc}
\hline Compound & $\begin{array}{c}\mathrm{MIC}_{99} \\
7 \mathrm{H} 9-\mathrm{ADC}\end{array}$ & $\mathrm{IC}_{50} \mathrm{CHO}$ & $\mathrm{GAST}-\mathrm{Fe}$ \\
\hline $\mathbf{1}[23]$ & $>125$ & $>125$ & $>10^{\#}$ \\
$\mathbf{2}[24]$ & $>125$ & $>125$ & $>322$ \\
$\mathbf{3}[19]$ & 82.20 & 82.8 & 119.00 \\
$\mathbf{4}[23]$ & $>125$ & $>125$ & $>254$ \\
$\mathbf{5}[23]$ & 125 & 80.9 & 84.80 \\
$\mathbf{6}[23]$ & 125 & $>125$ & $>254$ \\
$\mathbf{7}[18]$ & 68.70 & $>125$ & 9.54 \\
$\mathbf{8}[25]$ & $>125$ & $>125$ & $\mathrm{Nd}$ \\
$\mathbf{9}[26]$ & $>125$ & $>125$ & $>414$ \\
$\mathbf{1 0}[26]$ & $>125$ & $>125$ & $>349$ \\
$\mathbf{1 1}[27]$ & 50.00 & 42.8 & 7.40 \\
$\mathbf{1 2}[26]$ & $>125$ & $>125$ & $>386$ \\
$\mathbf{1 3}[28]$ & $>125$ & $>125$ & $>100^{+}$ \\
$\mathbf{1 4}[25]$ & 9.60 & 18.8 & 8.01 \\
$\mathbf{1 5}[25]$ & 13.90 & 15.2 & 8.20 \\
$\mathbf{1 6}$ & $>125$ & $>125$ & $>100^{+}$ \\
Rifampicin & 0.0149 & 0.0274 & $\mathrm{Nd}$ \\
Emetine & Nd & nd & 0.061 \\
\hline
\end{tabular}

$\mathrm{TB}: \mathrm{MIC}_{99} 7 \mathrm{H} 9(\mu \mathrm{M})$ in M. tuberculosis H37Rv:gfp. TB : MIC99 GAST-Fe $(\mu \mathrm{M})$ in M. tuberculosis H37Rv:gfp. CHO:Chinese hamster ovarian cell $\mathrm{IC}_{50}(\mu \mathrm{M})$ as indication of cytotoxicity $[18,19]$. nd: not determined. ${ }^{*}$ highest concentration measured. ${ }^{+}$CellTiter-Glo ${ }^{\circledR}$ luminescent cell viability assay [28].

compound 1, an oxapentacycloundecane benzyl amine derivative, and the azapentacycloundecane benzyl amine derivative, compound 5. Results point towards antimycobacterial activity being linked to the presence of the tertiary amine and/or free hydroxyl group within the polycyclic aza-cage. Compounds 11, 14, and 15 containing an adamantane moiety showed better activity compared to oxa- and aza-PCU-based compounds in general. These are also the only 3 compounds containing the 1,3-diamine linker versus the 1,2-diamine present in compounds 3, 6, and 7 . This increased activity could, therefore, be due to the presence of the adamantane or the 1,3-diamine linker. Comparable activity (Figure 2 ) seen in compounds 2 (oxaPCU) and 10 (adamantane derivative), however, suggest that the increased activity may be linked to the 1,3-diamine linker rather than the presence of the adamantane moiety.

2.2. Cytotoxicity Analysis. Cytotoxicity of the compounds was evaluated using a Chinese hamster ovarian $(\mathrm{CHO})$ cell line (Table 1). The marked cytotoxicity differences observed between compounds $\mathbf{3}$ and $\mathbf{1 1}$ were noteworthy. The adamantane moiety may contribute to increased cytotoxicity, but, as can be deduced from the similar $\mathrm{IC}_{50}$ values of compounds 2 and 10, it appears that this molecular feature cannot be solely responsible for the increased cytotoxicity observed for compound 11. The propane-1,3-diamine linker, however, seems to contribute to cytotoxicity as compounds 11, 14, and 15 all demonstrate significantly higher cytotoxicity, even when compared to most compounds containing the similar ethane-1,2-diamine moiety. The 1,3-diamine linker unfortunately also seems to play a role in antimycobacterial activity as these compounds demonstrate the lowest overall MIC values.

2.3. Preliminary Mechanism of Action Determination. To explore the potential MOA of the compounds, a number of 


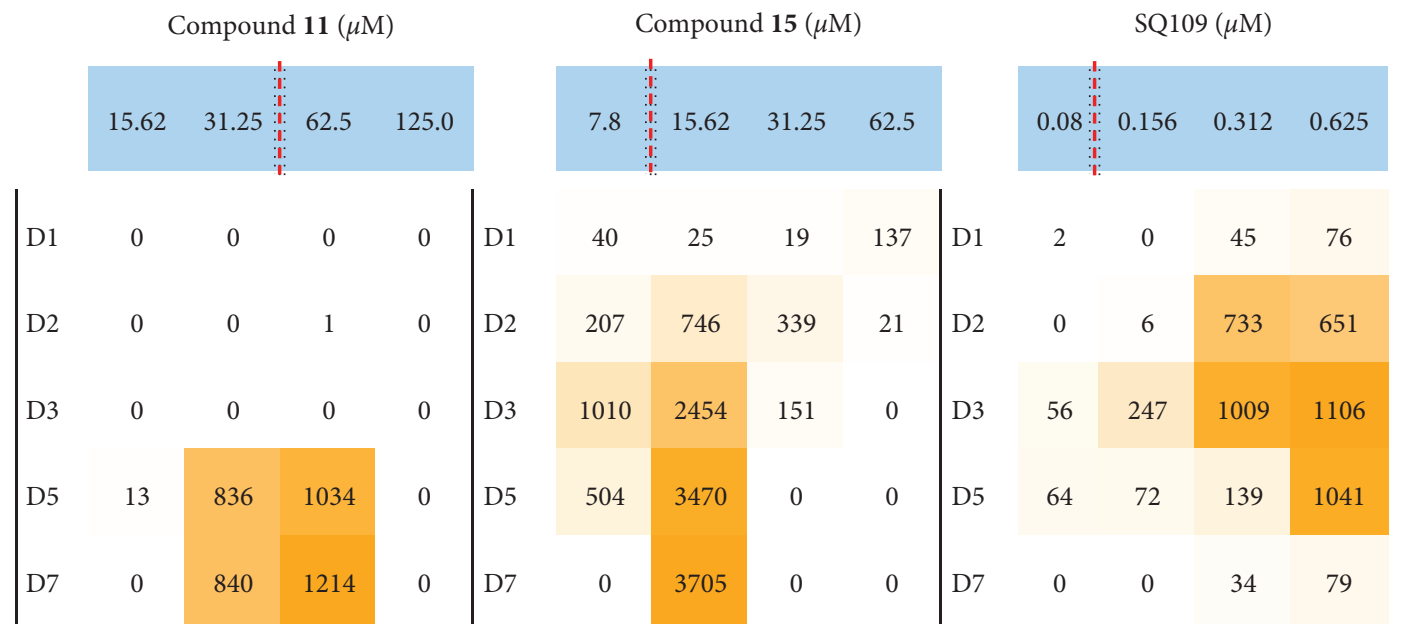

FIGURE 2: Observed luminescence data of compounds 11, 15, and SQ109 depicting cell wall damage over 7 days (D1-D7) at various concentration ranges $(\mu \mathrm{M})$ spanning the $\mathrm{MIC}_{90}$ (as indicated by the red dotted line).

bioactivity reporter assays were utilized. To evaluate the possible impact of the compounds on respiration, comparative MIC values were determined for the respective compounds in wild-type $M$. tuberculosis $\mathrm{H} 37 \mathrm{Rv}$ vs. (i) a cytochrome $b d$ oxidase deficient mutant $(\Delta c y d \mathrm{KO})$ and (ii) a $\Delta c y d \mathrm{KO} / \mathrm{qcrBA} 317 \mathrm{~T}$ strain to determine possible QcrB inhibition [33,34]. QcrB inhibitors are expected to exhibit increased potency (lower MIC) in the $\Delta c y d \mathrm{KO}$ but minimal activity in the $\Delta c y d \mathrm{KO} / q c r B \mathrm{~A} 317 \mathrm{~T}$ strain which carries an additional Ala317Thr point mutation in QcrB [35]. No significant shift in MIC was observed for any of the compounds against either of the mutant strains, strongly suggesting that none was likely to target QcrB $[34,36]$.

Next, a real-time bioluminescence assay [37] was used to investigate cell wall damage as a potential mechanism of action. Compounds with inhibitory effects on mycobacterial arabinogalactan, mycolic acid, and fatty acid synthesis have been shown to result in the upregulation of the iniBAC operon [38]. This operon is, however, not upregulated by other cell wall stressors, for example, exposure to hydrogen peroxide, heat, acidic conditions, or antibiotics that do not directly inhibit cell wall synthesis (e.g., aminoglycosides, fluoroquinolones, or rifampins) [38]. Compounds 14 and 15 were PiniB-LUX positive and showed early (day 1) and sustained luminescence signals, similar to the luminescence profile which was observed previously for ethambutol [37]. Figure 2 provides an example of bioluminescence observed for late and indirect cell wall damage (compound 11), early cell wall damage (compound 15), and the positive control SQ109 (also demonstrating early cell wall damage). Comparative background-corrected RLU data are presented as heat maps, ranging from white (minimum) to orange (maximum) as a function of the maximum RLU recorded for luminescence control.

Given the presence of the adamantane and amine linker functional groups in SQ109 [39], it was decided to use SQ109 as a control compound in the PiniB-LUX assay. SQ109 also demonstrated early luminescence, but the signal was only sustained for approximately 5 days [37], whereas in the current study, a continued signal was observed for compounds 14 and 15 over the course of the 14-day assay. Compounds 3, 7, and 11 were associated with delayed production of bioluminescence. This suggests that the impact on cell wall homeostasis might be delayed or a secondary effect of a compound with polypharmacologic activity. These data suggest that compounds 14 and 15 directly target cell wall biosynthesis, although the precise target is still unknown.

Finally, we investigated whether any of the compounds were genotoxic using the PrecA-LUX bioluminescence reporter [37]. RecA is a key regulator of mycobacterial DNA damage response and is induced after the exposure to compounds which are directly genotoxic (i.e., altering or damaging to the nucleic acid) or which inhibit DNA metabolism (replication and/or repair). Placing the bacterial luciferase luxCABE cassette under the transcriptional control of the recA promoter results in increased bioluminescence following promoter induction in response to DNA damage [37]. None of the compounds evaluated in this study produced a positive luminescence signal over the full duration of the assay, eliminating DNA damage as potential MOA.

2.4. Synergism Evaluations. It was originally postulated that compounds from this series might act as mycobacterial efflux pump inhibitors (EPIs). As an initial screen to determine possible EPI activity, synergism assays were performed using the known Rv1258c efflux pump substrate, spectinomycin, as an anchor compound. Spectinomycin is an aminocyclitol antibiotic with a unique binding site on the bacterial $30 \mathrm{~S}$ ribosomal subunit which affords it selective toxicity and a good side effect profile. Unfortunately, mainly due to extensive efflux by bacterial efflux pumps (specifically Rv1258c in M. tuberculosis), spectinomycin lacks significant antibacterial activity $[15,40,41]$. The activity of spectinomycin is increased substantially under conditions where efflux pump activity is inhibited [42] making it a useful agent 


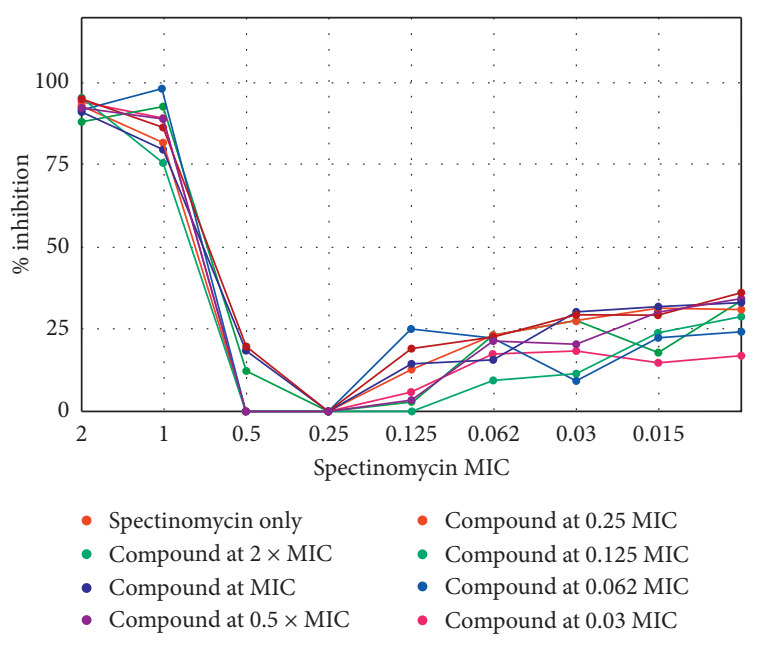

(a)

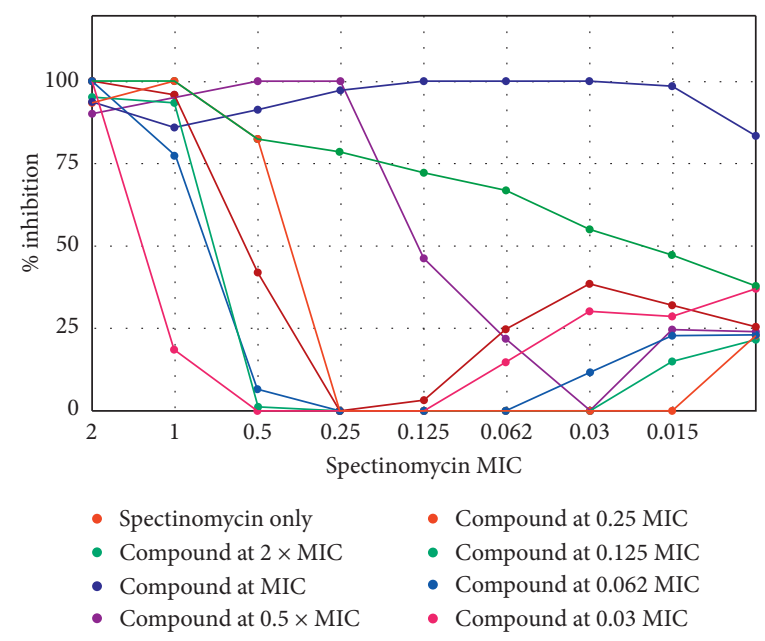

(b)

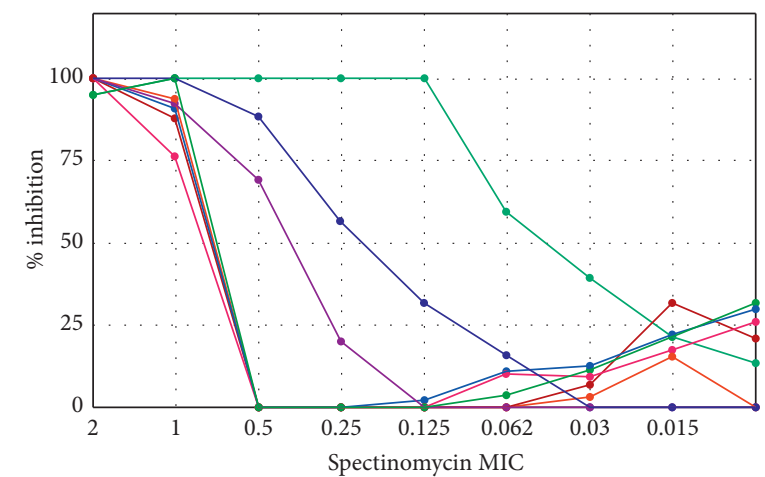

- Spectinomycin only

- Compound at $2 \times$ MIC

- Compound at MIC

- Compound at $0.5 \times$ MIC
- Compound at 0.25 MIC

- Compound at $0.125 \mathrm{MIC}$

- Compound at $0.062 \mathrm{MIC}$

- Compound at $0.03 \mathrm{MIC}$

(c)

FIgURE 3: Percentage inhibition of compound $\mathbf{1}(\mathrm{a}), \mathbf{5}$ (b), and $\mathbf{6}$ (c) at various concentration combination points where the concentration of spectinomycin (as a function of MIC) is indicated on the $x$-axis combined with multiples of the MIC of the test compound as indicated by the colors in the legend.

to screen for possible efflux pump inhibitory activity using synergism assays. All compounds apart from compounds $\mathbf{1 4}$ and 15, which were excluded due to inherent antimycobacterial activity, were screened for synergism with spectinomycin using a checkerboard assay. To this end, serial dilutions of the test compound and spectinomycin were added to an $M$. tuberculosis culture in 7H9 media on the $y$ and $x$-axis of a 96-well plate. Bacterial growth was measured, and data were analyzed as described [43]. Compounds 5 and 6 showed evidence of synergism with spectinomycin, but no significant changes in MIC were observed with any of the other compounds. Inhibition of bacterial growth for compounds 1, 5, and $\mathbf{6}$ in combination with spectinomycin is shown in Figure 3. The concentration of spectinomycin as a fraction of its MIC (i.e., $2=2 \times$ MIC, $1=$ MIC, $0.5=$ half MIC etc.) is given on the $x$-axis, combined with various fractions of the MIC of the test compound as indicated by the colors in the graph legend. The bacterial inhibition as measured in the spectinomycin-only wells at various multiples of spectinomycin MIC is depicted as the dark red (top) legend on the graph. If spectinomycin is combined with a test compound with which it synergizes, the percentage bacterial inhibition remains high despite decreases in spectinomycin concentration. Thus, compound $\mathbf{1}$ (A) which is provided for purposes of comparison with structurally similar compound 5 shows no evidence of synergism, but bacterial inhibition at lower concentrations of spectinomycin when combined with compounds 5 (B) and $\mathbf{6}$ (C) point to synergistic activity.

Both compound $\mathbf{5}$ and $\mathbf{6}$ are aza-cage derivatives. Again, the differences between the activity of the structurally similar compounds $\mathbf{1}$ and 5 (Figure 1) are notable. Similar to what was seen with the antimycobacterial activity of these two compounds, the tertiary nitrogen and free hydroxyl group on the aza-cage are likely involved in the observed synergistic activity. Compounds 3 and 7 , contrariwise, appeared to increase the MIC of spectinomycin at higher concentrations (data not shown). This observation might point to reduced absorption of spectinomycin but would require 
further investigation to determine the mechanism of this possible antagonism.

\section{Conclusions}

In this study, we explored possible antimycobacterial activity, mechanism of action, and synergistic activity of a series of polycyclic amine derivatives. The compounds were selected based on the presence of pharmacophoric moieties with possible antimycobacterial activity, previously described resistance reversal activity, as well as for the presence of structural features and bioactivity that may increase the accumulation of active moieties and coadministered antimycobacterial drugs within $M$. tuberculosis. The most active compound (15) demonstrated an $\mathrm{MIC}_{99}$ of $9.6 \mu \mathrm{M}$ against M. tuberculosis $\mathrm{H} 37 \mathrm{Rv}$ and will serve as a lead compound to improve selective antimycobacterial activity.

Inhibition of the $b c_{1}$ respiratory complex and DNA functionality as MOA as well as genotoxicity were excluded for the series. Cell wall damage, however, was identified as the likely mechanism of action of a number of compounds. Particularly, compounds $\mathbf{1 4}$ and $\mathbf{1 5}$ were shown to cause early and sustained cell wall damage in a fashion similar to ethambutol. Compounds 3, 7, and 11 showed delayed cell wall damage which may indicate cell wall stress as a secondary effect to interference with other cell systems or a delayed effect on cell wall integrity.

Compounds 5 and $\mathbf{6}$ showed evidence of synergistic activity with spectinomycin and thus possible efflux pump (specifically Rv1258c) inhibition.

The azapentacycloundecane cage seems to play a role in the antimycobacterial activity in nutrient poor media as well as the ability to synergize with spectinomycin as is evident from the large variances between the structurally similar compounds $\mathbf{1}$ and $\mathbf{5}$ in the respective assays. The role of the aza-cage in antimycobacterial and synergistic activity thus warrants further exploration. Although compounds from this series do not display sufficiently selective toxicity in single-drug in vitro assays, the ability of selected compounds from this series to act as chemosensitizers, may reduce the required MIC in combination therapy. Results from this preliminary study have contributed to our understanding of the structure activity relationships, possible synergistic activity, and toxicity for this series of compounds and will be used in conjunction with ongoing synergism assays to inform lead modification. Additional work exploring structure activity relationships for antimycobacterial activity (versus cytotoxicity) and, importantly, investigations pertaining to possible resistance reversal activity are underway in our laboratories.

\section{Materials and Methodology}

4.1. Minimum Inhibitory Concentration Determination. All assays were performed in a Biosafety Level III certified facility. Mycobacterium tuberculosis pMSp12:GFP [29, 44] was grown to an optical density (OD) of 0.6-0.7, and assays were performed using either GAST-Fe media $[45,46]$ or $7 \mathrm{H} 9$ media supplemented with 10\% Albumin Dextrose Catalase
(ADC) $[45,46] .10 \mathrm{mM}$ stock solutions of the test compounds in DMSO were serially diluted 2-fold, and plates were set up as previously described [47]. Rifampicin at $2 \mathrm{xMIC}$ was used as a minimum growth control. Relative fluorescence was measured and used to calculate the MIC as previously reported $[22,48]$.

4.2. Cytotoxicity Analysis. The reduction of 3-[4,5-dimethylthiazol-2-yl]-2,5-diphenyl tetrazolium bromide (MTT) in the presence of living cells was used to evaluate cell viability [49]. $20 \mathrm{mg} / \mathrm{mL}$ stock solutions of the test compounds in $10 \%$ DMSO were serially diluted in a complete medium with 10 -fold dilutions to obtain 6 concentrations. Cells were exposed to the respective test compounds for 44 hours after which MTT was added and cells were incubated at $37^{\circ} \mathrm{C}$ for a further 4 hours. Plates were analyzed at $540 \mathrm{~nm}$ using a spectrophotometer with wells containing untreated cells and growth medium only used to determine $100 \%$ survival and $0 \%$ survival, respectively. Emetine was used as a positive control, and assays were performed in triplicate. Cell viability was not compromised at the highest concentration of DMSO to which the cells were exposed (data not shown). $\mathrm{IC}_{50}$ values were obtained from full dose-response curves, using a nonlinear dose-response curve fitting analysis via GraphPad Prism v.4 software.

4.3. Respiratory Reporter Assay. Mycobacterium tuberculosis $\mathrm{H} 37 \mathrm{Rv} \Delta$ cydKO and Mycobacterium tuberculosis $\mathrm{H} 37 \mathrm{Rv}$ $\Delta$ cydKO/QcrBA317T $[34,35]$ were utilized as described above for the determination of the minimum inhibitory concentration (Section 4.1).

4.4. LUX Assays. A modified bioluminescence (LUX) reporter assay was used to investigate cell wall damage and genotoxicity as possible mechanisms of action [37]. A $10 \mathrm{ml}$ culture of the relevant Mycobacterium tuberculosis $\mathrm{H} 37 \mathrm{Rv}$ strain (PiniB-LUX determining cell wall damage or PrecALUX determining DNA damage) was grown to an optical density $\left(\mathrm{OD}_{600}\right)$ of \pm 0.4 . The strain culture was diluted $1: 10$ prior to inoculation of the assay. Two-fold serial dilutions of the test compounds and the assay control drugs were prepared in flat-bottomed 96-well microtiter plates. The relevant diluted $M$. tuberculosis reporter culture was added to each well to a final volume of $100 \mu \mathrm{l}$ per well. SQ109 and levofloxacin were used as a positive and negative control, respectively, in the PiniB-LUX assay, whereas levofloxacin and ethambutol were utilized as positive and negative control, respectively, in the PrecA-LUX assay.

Raw luminescent data (Relative Luminescence Units) were acquired using a SpecraMax i3x Plate reader (Molecular Devices Corporation, 1311 Orleans Drive, Sunnyvale, California 94089). Data were corrected for background luminescence using Softmax ${ }^{\circledR}$ Pro 6 software (Version 6.5.1, Serial no. 1278552768867612530), Molecular Devices Corporation, 1311 Orleans Drive, Sunnyvale, California 94089. Data were converted to 2-colour 2-dimensional heat maps 
using Microsoft ${ }^{\circledR}$ Excel $^{\circledR}$ for Mac 2011, Version 14.6.5 (160527), latest installed update 14.6.5.

4.5. Synergism Assays. Possible drug interactions of the various test compounds and spectinomycin were investigated utilizing a slightly modified standard two-dimensional (2D) checkerboard assay [42]. Serially diluted drugs were dispensed along the $x$-axis and $y$-axis of 96-well microtiter plates, respectively, at a starting concentration 100 times the final concentration. The assays were performed as previously described [42], and results were reported as a percentage growth inhibition at various drug concentration combinations.

\section{Data Availability}

Data are available on request from the corresponding author through e-mail (ekapp@uwc.ac.za).

\section{Conflicts of Interest}

The authors declare no conflicts of interest.

\section{Acknowledgments}

The authors thank the National Research Foundation of South Africa for providing financial support for this project (grant no. 117887). SLS received funding from the South African Research Chairs Initiative of the Department of Science and Technology and National Research Foundation (NRF) of South Africa, (award no. UID 86539). This project was also supported through the Strategic Health Innovation Partnerships (SHIP) Unit of the South African Medical Research Council with funds received from the South African Department of Science Innovation. The content is solely the responsibility of the authors and does not necessarily represent the official views of the NRF.

\section{References}

[1] M. Viveiros, M. Martins, L. Rodrigues et al., "Inhibitors of Mycobacterial efflux pumps as potential boosters for anti-tubercular drugs," Expert Review of Anti-Infective Therapy, vol. 10, pp. 983-998, 2012.

[2] J. P. Sarathy, V. Dartois, and E. J. D. Lee, “The role of transport mechanisms in Mycobacterium tuberculosis drug resistance and tolerance," Pharmaceuticals, vol. 5, no. 11, pp. 1210-1235, 2012.

[3] P. E. da Silva, A. V. Groll, A. Martin, and J. C. Palomino, "Efflux as a mechanism for drug resistance in Mycobacterium tuberculosis," FEMS Immunology and Medical Microbiology, vol. 63, no. 1, pp. 1-9, 2011.

[4] S. M. Batt, C. E. Burke, A. R. Moorey, and G. S. Besra, "Antibiotics and resistance: the two-sided coin of the mycobacterial cell wall," Cell Surface, vol. 6, Article ID 100044, 2020.

[5] K. Tahlan, R. Wilson, D. B. Kastrinsky et al., "SQ109 targets MmpL3, a membrane transporter of trehalose monomycolate involved in mycolic acid donation to the cell wall core of Mycobacterium tuberculosis," Antimicrobial Agents and Chemotherapy, vol. 56, no. 4, pp. 1797-1809, 2012.
[6] K. Hards, J. R. Robson, M. Berney et al., "Bactericidal mode of action of Bedaquiline," Journal of Antimicrobial Chemotherapy, vol. 70, no. 7, pp. 2028-2037, 2015.

[7] J. Zheng, E. J. Rubin, P. Bifani et al., "Para-aminosalicylic acid is a prodrug targeting dihydrofolate reductase in Mycobacterium tuberculosis," Journal of Biological Chemistry, vol. 288, no. 32, pp. 23447-23456, 2013.

[8] S. Chakraborty, T. Gruber, C. E. B. 3rd, H. I. Boshoff, and K. Y. Rhee, "Para-aminosalicylic acid acts as an alternative substrate of folate metabolism in Mycobacterium tuberculosis," Science, vol. 339, no. 6115, pp. 88-91, 2013.

[9] S. Chetty, M. Ramesh, A. Singh-Pillay, and M. E. S. Soliman, "Recent advancements in the development of anti-tuberculosis drugs," Bioorganic and Medicinal Chemistry Letters, vol. 27, no. 3, pp. 370-386, 2017.

[10] V. Dartois, "The path of anti-tuberculosis drugs: from blood to lesions to mycobacterial cells," Nature Reviews Microbiology, vol. 12, no. 3, pp. 159-167, 2014.

[11] S. M. Gygli, S. Borrell, A. Trauner, and S. Gagneux, "Antimicrobial resistance in Mycobacterium tuberculosis: mechanistic and evolutionary perspectives," FEMS Microbiology Reviews, Oxford University Press, vol. 41, , pp. 354-373, 2017.

[12] L. H. M. Te Brake, G. J. De Knegt, J. E. De Steenwinkel et al., "The role of efflux pumps in tuberculosis treatment and their promise as a target in drug development: unraveling the black box," Annual Review of Pharmacology and toxicology, vol. 58, pp. 271-291, 2018.

[13] D. Fange, K. Nilsson, T. Tenson, and M. Ehrenberg, "Drug efflux pump deficiency and drug target resistance masking in growing bacteria," Proceedings of the National Academy of Sciences of the United States of America, vol. 106, no. 20, pp. 8215-8220, 2009.

[14] F. P. Rodriguez-Rivera, X. Zhou, J. A. Theriot, and C. R. Bertozzi, "Visualization of Mycobacterial membrane dynamics in live cells," Journal of the American Chemical Society, vol. 139, no. 9, pp. 3488-3495, 2017.

[15] R. E. Lee, J. G. Hurdle, J. Liu et al., "Spectinamides: a new class of semisynthetic antituberculosis agents that overcome native drug efflux," Nature Medicine, vol. 20, no. 2, pp. 152-158, 2014.

[16] J. Jang, R. Kim, M. Woo et al., "Efflux attenuates the antibacterial activity of Q203 in Mycobacterium tuberculosis," Antimicrobial Agents and Chemotherapy, vol. 61, no. 7, 2017.

[17] J. Joubert, W. J. Geldenhuys, C. J. der Schyf et al., "Polycyclic cage structures as lipophilic scffolds for neuroactive drugs," ChemMedChem, vol. 7, no. 3, pp. 375-384, 2012.

[18] J. Joubert, E. E. Fortuin, D. Taylor, P. J. Smith, and S. F. Malan, "Pentacycloundecylamines and conjugates thereof as chemosensitizers and reversed chloroquine agents," Bioorganic and Medicinal Chemistry Letters, vol. 24, no. 23, pp. 55165519, 2014.

[19] J. Joubert, E. Kapp, D. Taylor, P. J. J. Smith, and S. F. F. Malan, "Polycyclic amines as chloroquine resistance modulating agents in plasmodium falciparum," Bioorganic and Medicinal Chemistry Letters, vol. 26, no. 4, pp. 1151-1155, 2016.

[20] E. Grobler, A. Grobler, C. J. der Schyf, and S. F. Malan, "Effect of polycyclic cage amines on the transmembrane potential of neuronal cells," Bioorganic and Medicinal Chemistry, vol. 14, no. 4, pp. 1176-1181, 2006.

[21] E. Kapp, S. F. Malan, J. Joubert, and S. L. Sampson, "Small molecule efflux pump inhibitors in Mycobacterium tuberculosis: a rational drug design perspective," Mini Reviews in Medicinal Chemistry, vol. 18, no. 1, pp. 72-86, 2018. 
[22] E. Kapp, H. Visser, S. L. Sampson et al., "Versatility of 7substituted coumarin molecules as antimycobacterial agents, neuronal enzyme inhibitors and neuroprotective agents," Molecules, vol. 22, no. 10, 2017.

[23] J. Joubert, R. Sharma, M. Onani, and S. F. Malan, "Microwaveassisted methods for the synthesis of pentacyclo [5.4.0.02,6.03,10.05,9] Undecylamines," Tetrahedron Letters, vol. 54, no. 50, pp. 6923-6927, 2013.

[24] S. F. Malan, J. V. der Walt, and C. V. der Schyf, "Structureactivity relationships of polycyclic mines with calcium channel blocking activity," Archiv der Pharmazie, vol. 333, pp. $10-16,2000$.

[25] H. J. Lemmer, J. Joubert, S. van Dyk, F. H. van der Westhuizen, and S. F. Malan, "S-nitrosylation and attenuation of excessive calcium flux by pentacycloundecane derivatives," Medicinal Chemistry, vol. 8, no. 3, pp. 361-371, 2012.

[26] Y. E. Kadernani, F. T. Zindo, E. Kapp, S. F. Malan, and J. Joubert, "Adamantane amine derivatives as dual acting NMDA receptor and voltage-gated calcium channel inhibitors for neuroprotection," Medchemcomm, vol. 5, no. 11, pp. 1678-1684, 2014.

[27] J. Joubert, S. van Dyk, I. R. Green, and S. F. Malan, "Synthesis and evaluation of fluorescent heterocyclic aminoadamantanes as multifunctional neuroprotective agents," Bioorganic and Medicinal Chemistry, vol. 19, no. 13, pp. 3935-3944, 2011.

[28] E. B. Foxen, Novel Cinnamic-Adamantane Conjugates as Potential Anti-denque and Anti-malaria Agents, 2012.

[29] G. L. Abrahams, A. Kumar, S. Savvi et al., "Pathway-selective sensitization of Mycobacterium tuberculosis for target-based whole-cell screening," Chemistry and Biology, vol. 19, no. 7, pp. 844-854, 2012.

[30] C. Changsen, S. G. Franzblau, and P. Palittapongarnpim, "Improved green fluorescent protein reporter gene-based microplate screening for antituberculosis compounds by utilizing an acetamidase promoter," Antimicrobial Agents and Chemotherapy, vol. 47, no. 12, pp. 3682-3687, 2003.

[31] S. G. Franzblau, R. S. Witzig, J. C. McLaughlin et al., "Rapid, low-technology MIC determination with clinical Mycobacterium tuberculosis isolates by using the microplate Alamar blue assay," Journal of Clinical Microbiology, vol. 36, no. 2, pp. 362-366, 1998.

[32] K. Pethe, P. C. Sequeira, S. Agarwalla et al., "A chemical genetic screen in Mycobacterium tuberculosis identifies carbon-source-dependent growth inhibitors devoid of in vivo efficacy," Nature Communications, vol. 1, p. 57, 2010.

[33] A. Moosa, D. A. Lamprecht, K. Arora et al., "Susceptibility of Mycobacterium tuberculosis cytochrome bd oxidase mutants to compounds targeting the terminal respiratory oxidase, cytochrome c," Antimicrobial Agents and Chemotherapy, vol. 61 , no. $10,2017$.

[34] K. Arora, B. Ochoa-Montaño, P. S. Tsang et al., "Respiratory flexibility in response to inhibition of cytochrome c oxidase in Mycobacterium tuberculosis," Antimicrobial Agents and Chemotherapy, vol. 58, no. 11, pp. 6962-6965, 2014.

[35] R. Van Der Westhuyzen, S. Winks, C. R. Wilson et al., "Pyrrolo[3,4-c]Pyridine-1,3(2H)-Diones: a novel antimycobacterial class targeting mycobacterial respiration," Journal of Medicinal Chemistry, vol. 58, no. 23, pp. 9371-9381, 2015.

[36] N. P. Kalia, E. J. Hasenoehrl, N. B. A. Rahman et al., "Exploiting the synthetic lethality between terminal respiratory oxidases to kill Mycobacterium tuberculosis and clear host infection,"
Proceedings of the National Academy of Sciences of the United States of America, vol. 114, no. 28, pp. 7426-7431, 2017.

[37] K. Naran, A. Moosa, C. E. Barry, H. I. M. Boshoff, V. Mizrahi, and D. F. Warner, "Bioluminescent reporters for rapid mechanism of action assessment in tuberculosis drug discovery," Antimicrobial Agents and Chemotherapy, vol. 60, no. 11, pp. 6748-6757, 2016.

[38] D. Alland, A. J. Steyn, T. Weisbrod, K. Aldrich, and W. R. Jacobs, "Characterization of the Mycobacterium tuberculosis IniBAC promoter, a promoter that responds to cell wall biosynthesis inhibition," Journal of Bacteriology, vol. 182, no. 7, pp. 1802-1811, 2000.

[39] M. Protopopova, C. Hanrahan, B. Nikonenko et al., "Identification of a new antitubercular drug candidate, SQ109, from a combinatorial library of 1,2-ethylenediamines," Journal of Antimicrobial Chemotherapy, vol. 56, no. 5, pp. 968-974, 2005, [pii].

[40] M. Balganesh, N. Dinesh, S. Sharma, S. Kuruppath, A. V. Nair, and U. Sharma, "Efflux pumps of Mycobacterium tuberculosis play a significant role in antituberculosis activity of potential drug candidates," Antimicrobial Agents and Chemotherapy, vol. 56, no. 5, pp. 2643-2651, 2012, [pii].

[41] J. Liu, D. F. Bruhn, R. B. Lee et al., "Structure-activity relationships of spectinamide antituberculosis agents: a dissection of ribosomal inhibition and native efflux avoidance contributions," ACS Infectious Diseases, vol. 3, no. 1, pp. 72-88, 2017.

[42] C. Omollo, V. Singh, E. Kigondu et al., "Developing synergistic drug combinations to restore antibiotic sensitivity in drug-resistant Mycobacterium Tuberculosis," Antimicrobial Agents and Chemotherapy, vol. 65, no. 5, Article ID 860288, 2021.

[43] K. Singh, M. Kumar, E. Pavadai et al., "Synthesis of new verapamil analogues and their evaluation in combination with rifampicin against Mycobacterium tuberculosis and molecular docking studies in the binding site of efflux protein Rv1258c," Bioorganic and Medicinal Chemistry Letters, vol. 24, no. 14, pp. 2985-2990, 2014.

[44] L. A. Collins, M. N. Torrero, and S. G. Franzblau, "Green fluorescent protein reporter microplate Assay for highthroughput screening of compounds against Mycobacterium tuberculosis," Antimicrobial Agents and Chemotherapy, vol. 42, no. 2, pp. 344-347, 1998.

[45] J. J. De Voss, K. Rutter, B. G. Schroeder, H. Su, Y. Zhu, and C. E. Barry, "The salicylate-derived mycobactin siderophores of Mycobacterium tuberculosis are essential for growth in macrophages," Proceedings of the National Academy of Sciences of the United States of America, vol. 97, no. 3, pp. 1252-1257, 2000.

[46] S. G. Franzblau, M. A. Degroote, S. H. Cho et al., "Comprehensive analysis of methods used for the evaluation of compounds against Mycobacterium Tuberculosis," Tuberculosis, vol. 92, no. 6, pp. 453-488, 2012.

[47] J. Ollinger, M. A. Bailey, G. C. Moraski et al., "A dual read-out assay to evaluate the potency of compounds active against Mycobacterium tuberculosis," PLoS One, vol. 8, no. 4, 2013.

[48] Collaborative Drug Discovery Inc. (CDD), "Collaborative drug discovery," Home-Collaborative Drug Discovery Inc. (CDD), 2020, https://www.collaborativedrug.com/de/.

[49] T. Mosmann, "Rapid colorimetric assay for cellular growth and survival: application to proliferation and cytotoxicity assays," Journal of Immunological Methods, vol. 65, no. 1-2, pp. 55-63, 1983. 